\title{
TIM-3 inhibits PDGF-BB-induced atherogenic responses in human artery vascular smooth muscle cells
}

\author{
CHONG LIAN $^{1}$, ZHECUN WANG $^{1}$, JIACONG QIU ${ }^{1}$, BAOHONG JIANG ${ }^{1}$, JUNBING LV ${ }^{1}$, \\ RONGZHOU HE ${ }^{1}$, RUIMING LIU ${ }^{1}$, WEN LI $^{2}$, JINSONG WANG ${ }^{1 *}$ and SHENMING WANG ${ }^{1 *}$ \\ ${ }^{1}$ Division of Vascular Surgery; ${ }^{2}$ Laboratory of General Surgery, \\ Guangdong Key Engineering Laboratory for Diagnosis and Treatment of Vascular Disease, \\ The First Affiliated Hospital, Sun Yat-sen University, Guangzhou, Guangdong 510080, P.R. China
}

Received October 3, 2019; Accepted April 7, 2020

DOI: $10.3892 / \mathrm{mmr} .2020 .11167$

\begin{abstract}
Increasing evidence suggests that T-cell immunoglobulin and mucin domain 3 (TIM-3) displays anti-atherosclerotic effects, but its role in vascular smooth muscle cells (VSMCs) has not been reported. The present study aimed to investigate the function of TIM-3 and its roles in human artery VSMCs (HASMCs). A protein array was used to investigate the TIM-3 protein expression profile, which indicated that TIM-3 expression was increased in the serum of patients with lower extremity arteriosclerosis obliterans disease (LEAOD) compared with healthy individuals. Immunohistochemistry and western blotting of arterial tissue further revealed that TIM-3 expression was increased in LEAOD artery tissue compared with normal artery tissue. Additionally, platelet-derived growth factor-BB (PDGF-BB) displayed a positive correlation with TIM-3 expression in HASMCs. TIM-3 decreased the migration and proliferation of PDGF-BB-induced HASMCs, and anti-TIM-3 blocked the effects of TIM-3. The effect of TIM- 3 on the proliferation and migration of HASMCs was further investigated using LV-TIM-3-transduced cells. The results revealed that TIM-3 also inhibited PDGF-BB-induced expression of the inflammatory factors interleukin- 6 and tumor necrosis factor- $\alpha$ by suppressing NF- $\mathrm{\kappa B}$ activation. In summary, the present study revealed that TIM-3 displayed a regulatory role during the
\end{abstract}

Correspondence to: Dr Jinsong Wang or Dr Shenming Wang, Division of Vascular Surgery, Guangdong Key Engineering Laboratory for Diagnosis and Treatment of Vascular Disease, The First Affiliated Hospital, Sun Yat-sen University, 58 Zhong Shan Er Road, Guangzhou, Guangdong 510080, P.R. China

E-mail: wangjs@mail.sysu.edu.cn

E-mail: shenmingwang@hotmail.com

${ }^{*}$ Contributed equally

Key words: T-cell immunoglobulin and mucin domain 3, migration, proliferation, atherosclerosis, inflammation, platelet-derived growth factor-BB, human artery vascular smooth muscle cells
PDGF-BB-induced inflammatory reaction in HASMCs, which indicated that TIM-3 may display anti-atherosclerotic effects.

\section{Introduction}

Lower extremity arteriosclerosis obliterans disease (LEAOD) affects $\sim 17 \%$ of individuals aged 55-75 years worldwide, and is characterized by intermittent claudication, rest pain and gangrene caused by lower extremity ischemia $(1,2)$. Previous studies have indicated that atherosclerosis is a persistent chronic inflammatory disease $(3,4)$. The initial steps of atherosclerosis formation are endothelial damage, followed by platelet aggregation and leukocyte infiltration under the injured endothelial cells. Subsequently, macrophage activation, smooth muscle cell proliferation and migration, and foam cell formation occur, eventually leading to atherosclerosis $(5,6)$.

Platelet-derived growth factor-BB (PDGF-BB) serves an important role in the initiation and progression of atherosclerosis (7). The expression of PDGF-BB in vascular smooth muscle cells (VSMCs) is upregulated and activated in injured vessels (7). Previous studies have reported that PDGF-BB can induce a variety of biological effects by increasing the expression of proinflammatory cytokines, such as interleukin (IL)-1 $\beta$, IL-17A and TNF- $\alpha$, which can induce the proliferation and migration of VSMCs $(8,9)$. Therefore, inhibiting the proliferation and migration of VSMCs caused by PDGF-BB may serve as an important preventative strategy for atherosclerosis.

A number of proteins, including tumor necrosis factor (TNF) superfamily member 11 (TRANCE) (10), IL-6 (11) and transforming growth factor (TGF)- $\beta(12,13)$, serve potentially important roles during the development and progression of atherosclerosis. T-cell immunoglobulin and mucin domain 3 (TIM-3) as a regulatory factor alters the function of a number of different immune cells, including effector/regulatory $\mathrm{T}$ cells, macrophages, monocytes and NK cells $(14,15)$. Previous studies using immune cell experimental models have identified TIM-3 as an antiatherosclerotic agent during atherosclerosis progression $(16,17)$.

However, the regulatory role of TIM-3 onPDGF-BB-induced human artery vascular smooth muscle cell (HASMC) proliferation and migration, and how the pathway is activated remains 
unclear. In the present study, the effects of TIM-3 on HASMCs and the underlying mechanisms were investigated.

\section{Materials and methods}

Specimen collection. Serum samples and femoral arterial specimens were collected in at The First Affiliated Hospital of Sun Yat-sen University, China between September 2017 and July 2019. Serum samples were collected from five patients with LEAOD with the mean age of 63.5 years old (four male and one female; age range: 56-75 years old) and five healthy controls with the mean age of 65.5 years old (four male and one female; age range: 52-71 years old). Serum samples of the two groups were also age, sex and ethnicity-matched. Serum samples were stored at $-80^{\circ} \mathrm{C}$ until further analysis. Femoral arterial specimens were collected from five patients with LEAOD with the mean age of 62 years old (three male and two female; age range: 53-73 years old) who had undergone amputations due to severe lower extremity ischemia and gangrene in the vascular surgery department, and normal femoral arterial specimens were collected from five cases without LEAOD with the mean age of 60.5 years old (four male and one female; age range: 50-69 years old) who had undergone amputations due to severe lower limb trauma caused by a traffic accident in the departments of trauma surgery and emergency. Internal and external tissues were removed from the blood vessels during specimen collection. After washing with normal saline, some specimens were immediately frozen in liquid nitrogen and some specimens were fixed in $4^{\circ} \mathrm{C}, 4 \%$ formaldehyde until further analysis. Written informed consent was obtained from the participants or their relatives. The present study was approved by the Research Ethics Committee of the First Affiliated Hospital of Sun Yat-sen University.

Protein arrays. Serum samples were analyzed using the Human Cytokine Antibody Array 440 (cat. no. QAH-CAA-440; RayBiotech, Inc.), according to the manufacturer's protocol.

Cell culture and treatment. HASMCs were isolated from the femoral artery specimens and identified by anti- $\alpha$-smooth muscle actin antibody staining as previously described (18). HASMCs were cultured in SMCM medium (Sciencell Research Laboratories, Inc.) supplemented with 10\% FBS (Gibco; Thermo Fisher Scientific, Inc.) at $37^{\circ} \mathrm{C}$ in a $5 \% \mathrm{CO}_{2}$ humidified incubator. HASMCs from passages 5 to 9 were used for subsequent experiments. When the cell density reached $50-60 \%$, HASMCs were incubated with $20 \mathrm{ng} / \mathrm{ml}$ PDGF-BB (R\&D Systems, Inc.), TIM-3 (1,000 ng/ml; R\&D Systems, Inc.), anti-TIM-3 mAb (10 $\mu \mathrm{g} / \mathrm{ml}$; BioLegend, Inc.) or were only incubated with serum supplemented with 10\% FBS (Gibco; Thermo Fisher Scientific, Inc.) at $37^{\circ} \mathrm{C}$ for $48 \mathrm{~h}$. Subsequently, HASMCs were transduced with the LV-TIM-3 lentiviral vector (Genechem Co., Ltd.) according to the manufacturer's protocol The empty $\mathrm{LV}$-vector (Genechem Co., Ltd.) was used as the control.

$E d U$ assay. HASMCs were treated according to the manufacturer's protocol (Cell-Light EdU Apollo567 Kit; Guangzhou RiboBio Co., Ltd.). EdU-positive cells were observed using an Axio Observer Z1 fluorescence microscope (Zeiss GmbH) and analyzed using ImageJ version 1.8.0 (National Institutes of Health).
Transwell and wound-healing assays. For the Transwell assay, Treated HASMCs ( $1 \times 10^{4}$ cells/well) resuspended in serum-free SMCM were plated into the upper chambers of the Transwell plates ( 24 wells; $\mathrm{dr}=6.5 \mathrm{~mm}$; pore size: $8.0 \mu \mathrm{m}$; Corning, Inc.) and the lower chambers were plated in 10\% FBS SMCM. After incubation at $37^{\circ} \mathrm{C}$ for $24 \mathrm{~h}$, migratory cells on the lower surface of the Transwell membranes were fixed using $4 \%$ formaldehyde at $25^{\circ} \mathrm{C}$ for $20 \mathrm{~min}$ and washed twice with PBS. Then the membranes were stained with $0.1 \%$ crystal violet at $25^{\circ} \mathrm{C}$ for $15 \mathrm{~min}$.

For the wound-healing assay, at $\sim 90 \%$ confluency, a $1,000-\mu 1$ disposable pipette tip was used to form a straight scratch wound through the treated HASMC cell monolayer. The cell layer was washed with PBS gently and subsequently incubated with serum-free SMCM medium. Images of the wound were captured using a light microscope x100 magnification (BX51W; Olympus Co., Ltd.) at 0 and $24 \mathrm{~h}$. Cell migration was analyzed using ImageJ Version 1.8.0 (National Institutes of Health).

Western blot analysis. HASMCs were washed with precooled PBS for three times and subsequently total protein was extracted from HASMCs using RIPA buffer (Thermo Fisher Scientific, Inc.) on ice. Then the protein concentration was quantified by bicinchoninic acid method. Equal amounts of protein $(20 \mu \mathrm{g} / \mathrm{lane})$ were separated by $10-15 \%$ SDS-PAGE, and then transferred to PVDF membranes (EMD Millipore). Subsequently, the membranes were blocked with $5 \%$ bovine serum albumin (BioFroxx, Inc.) at $22-25^{\circ} \mathrm{C}$ for $1 \mathrm{~h}$. Then the membranes were incubated overnight at $4^{\circ} \mathrm{C}$ with primary antibodies targeted against: TIM-3 (1:500; cat. no. 1701-20; Hangzhou HuaAn Biotechnology Co., Ltd.), PDGF-BB (1:500; cat. no. 9704; Abcam), p65 NFkB (1:1,000; cat. no. 8242; Cell Signaling Technology, Inc.), phosphorylated (p)-p65 NFкB (1:1,000; cat. no. 3033; Cell Signaling Technology, Inc.), IL-6 (1:1,000; cat. no. 12153; Cell Signaling Technology, Inc.), TNF- $\alpha$ (1:1,000; cat. no. 8184; Cell Signaling Technology, Inc.) and GAPDH (1:1,000; cat. no. 5174; Cell Signaling Technology, Inc.). Following primary incubation, the membranes were incubated at $22-25^{\circ} \mathrm{C}$ for $1 \mathrm{~h}$ with corresponding rabbit anti-mouse horseradish peroxidase(HRP)-conjugated secondary antibody (1:3,000; cat. no. 58802, Cell Signaling Technology, Inc.) and mouse anti-rabbit HRP-conjugated secondary antibody (1:5,000; cat. no. 93702; Cell Signaling Technology, Inc.). Protein bands were visualized using the Novex ECL kit (Invitrogen; Thermo Fisher Scientific, Inc.). Protein expression was quantified using ImageJ Version 1.8.0 (National Institutes of Health) with GAPDH as the loading control.

Flow cytometry. Flow cytometry was performed to detect the quantitative proportion of TIM-3-positive HASMCs. Forward scatter (FSC) and side scatter (SSC) were used to find viable, single cell populations. Treated HASMCs were resuspended and labeled with the TIM-3 (D5D5R ${ }^{\mathrm{TM}}$ ) Rabbit mAb (PE Conjugate) (cat. no. 67761, Cell Signaling Technology, Inc.) Unstained HASMCs were used to set the negative control populations. TIM-3-positive cells were then determined from this gated population by flow cytometry using the CyAN ${ }^{\mathrm{TM}}$ ADP flow cytometer (Beckman-Coulter, Inc.) and analyzed by FlowJo Version 10.0 (Tree Star, Inc.). 
Immunohistochemistry. Femoral arterial tissues were fixed in $4 \%$ polyformaldehyde at $22-25^{\circ} \mathrm{C}$ for $24 \mathrm{~h}$ and then embedded in paraffin. Following deparaffinization, rehydration with a descending alcohol series, $3 \%$ hydrogen peroxide was used to block the endogenous peroxidase activity, then the citric acid buffer was used for antigen retrieval at $95^{\circ} \mathrm{C}$ for $10 \mathrm{~min}$ and PBS buffer was used for washing. The sections $(4 \mu \mathrm{m})$ were incubated at $4^{\circ} \mathrm{C}$ overnight with anti-TIM-3 (1:100; cat. no. 1701-20; Hangzhou HuaAn Biotechnology Co., Ltd.). Subsequently, the sections were incubated at room temperature for $1 \mathrm{~h}$ with goat anti-mouse IgG-PE: (1:200; cat. no. sc-3738; Santa Cruz Biotechnology, Inc.). The sections were counterstained with hematoxylin at $22-25^{\circ} \mathrm{C}$ for $5 \mathrm{~min}$ and observed using a light microscope (BX51W1; Olympus Corporation) at $\mathrm{x} 400$ magnification. The images were analyzed using ImageJ Version 1.8.0 (National Institutes of Health).

Statistical analysis. Data are presented as the mean \pm SEM. The Student's t-test was used to compare data containing two groups. One-way ANOVA followed by Tukey's post hoc test was used to compare data containing $\geq 3$ groups. Pearson's correlation coefficient test was used to analyze the correlation between two indexes. Statistical analyses were performed using SPSS software (version 17.0; SPSS, Inc.). P $<0.05$ was considered to indicate a statistically significant difference.

\section{Results}

Microarray comparison of the protein expression profiles in the serum between patients with LEAOD and healthy controls. To further investigate the mechanisms underlying LEAOD, a 440-protein array was used to analyze the differential expression of proteins in the serum of five patients with LEAOD compared with healthy controls. A total of 22 proteins were differentially expressed, 13 proteins were upregulated and 9 proteins were downregulated in the serum of patients with LEAOD compared with healthy controls. The top 10 most highly expressed proteins were TRANCE, TIM-3, CD6, TGF- $\beta$, troponin I, IL- $1 \beta$, P-cadherin, TNF-related apoptosis-inducing ligand receptor-3 (TRAIL R3), TNF factor-like weak inducer of apoptosis receptor (TWEAK) and vascular cell adhesion molecule-1 (VCAM-1) (Table I).

Increased expression of TIM-3 in LEAOD arterial tissues. According to the immunohistochemistry results, the tunica media of LEAOD arterial tissues exhibited high levels of TIM-3 positive staining, whereas normal arterial tissues exhibited little TIM-3 positive staining (Fig. 1A). The protein array data for TIM-3 were further investigated using western blotting. TIM-3 and PDGF-BB expression levels were significantly higher in LEAOD arterial tissues compared to normal arterial tissues (Fig. 1B). A positive correlation between the relative expression PDGF-BB and TIM-3 was also identified (Fig. 1C).

TIM-3 inhibits proliferation and migration in PDGF-BB-stimulated HASMCs. Previous studies have reported that the expression of TIM-3 is upregulated in atherosclerotic patients or animal models $(16,19,20)$. In the present study, it was demonstrated that the expression of TIM-3 was upregulated in LEAOD arterial tissues (Fig. 1B). It is likely that the expression of
Table I. Top 10 most highly expressed proteins in the serum of patients with lower extremity arteriosclerosis obliterans disease vs. healthy control.

\begin{tabular}{clcc}
\hline Rank & Protein & Fold-change & P-value \\
\hline 1 & TRANCE & 3.12 & $0.012^{\mathrm{a}}$ \\
2 & TIM-3 & 2.38 & $0.027^{\mathrm{a}}$ \\
3 & CD6 & 2.16 & $0.011^{\mathrm{a}}$ \\
4 & TGF- $\beta$ & 1.66 & $0.044^{\mathrm{a}}$ \\
5 & Troponin I & 1.59 & $0.022^{\mathrm{a}}$ \\
6 & IL-1 $\beta$ & 1.17 & $0.010^{\mathrm{a}}$ \\
7 & P-cadherin & 1.13 & $0.011^{\mathrm{a}}$ \\
8 & TRAIL R3 & 1.06 & $0.027^{\mathrm{a}}$ \\
9 & TWEAK & 0.95 & $0.018^{\mathrm{a}}$ \\
10 & VCAM-1 & 0.72 & $0.015^{\mathrm{a}}$ \\
\hline
\end{tabular}

${ }^{\mathrm{a}} \mathrm{P}<0.05$. TNF, tumor necrosis factor; TRANCE, tumor necrosis factor superfamily member 11; TIM-3, T-cell immunoglobulin and mucin domain 3 ; TGF- $\beta$, transforming growth factor- $\beta$; IL- $1 \beta$, interleukin-1 $\beta$; TRAIL R3, TNF-related apoptosis-inducing ligand receptor-3; TWEAK, TNF-like weak inducer of apoptosis receptor; VCAM-1, vascular cell adhesion molecule-1.

TIM-3 is upregulated in atherosclerotic VSMCs, due to there are high numbers of VSMCs are in the media of arterial tissues (21). PDGF-BB is a critical factor that promotes the migration and proliferation of VSMCs, which causes atherosclerosis (7). HASMCs were stimulated with various concentrations of PDGF-BB. A dose-dependent relationship was identified between PDGF-BB concentration and TIM-3 expression by HASMCs (Fig. 2A). EdU (Fig. 2B), Transwell (Fig. 2C) and wound healing (Fig. 2D) assays were performed to assess HASMC proliferation and migration following stimulation with PDGF-BB $(20 \mathrm{ng} / \mathrm{ml})$ in the absence or presence of TIM-3 (1,000 ng/ml) and anti-TIM-3 $\mathrm{mAb}(10 \mu \mathrm{g} / \mathrm{ml})$. The proportion of EdU-positive cells was significantly higher in the PDGF-BB group $(30.36 \pm 3.02 \%)$ compared to the control group $(12.64 \pm 1.71 \%)$. Furthermore, the proportion of EdU-positive cells was significantly higher in the PDGF-BB + anti-TIM-3 group (40.54 $\pm 3.24 \%)$ compared to the PDGF-BB group $(30.36 \pm 3.02 \%)$. However, the proportion of EdU-positive cells was significantly reduced in the PDGF-BB + TIM-3 group compared to the PDGF-BB and PDGF-BB + anti-TIM-3 groups, but was not significantly different to the control group (Fig. 2B). Treatment with PDGF-BB significantly increased the mean number of migratory cells compared to the control group (69.6 \pm 7.4 vs. $26.2 \pm 5.6)$. The PDGF-BB + anti-TIM-3 group exhibited a significantly increased mean number of migratory cells compared to the PDGF-BB group $(93.0 \pm 5.5$ vs. $69.6 \pm 7.4)$. However, the PDGF-BB + TIM-3 group exhibited significantly decreased migratory ability compared to the PDGF-BB and PDGF-BB + anti-TIM-3 groups, and a similar level compared to the control group (Fig. 2C). The wound healing assay results were similar to the Transwell assay results (Fig. 2D). The results indicated that PDGF-BB promoted the proliferation and migration of HASMCs, TIM-3 inhibited the effect of PDGF-BB on HASMCs, and anti-TIM-3 blocked the effect of TIM-3 to promote the proliferation and migration of HASMCs. 
A
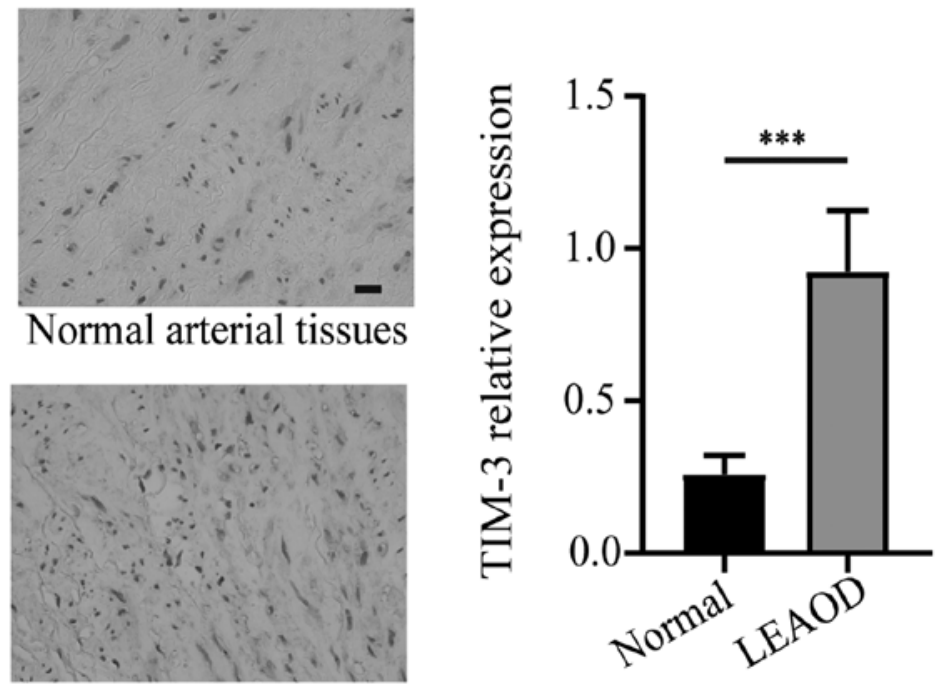

LEAOD arterial tissues

$\mathrm{B}$

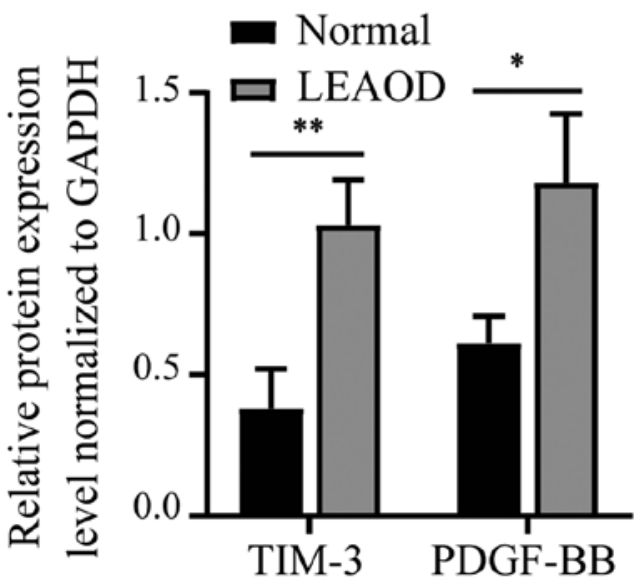

$\mathrm{C}$

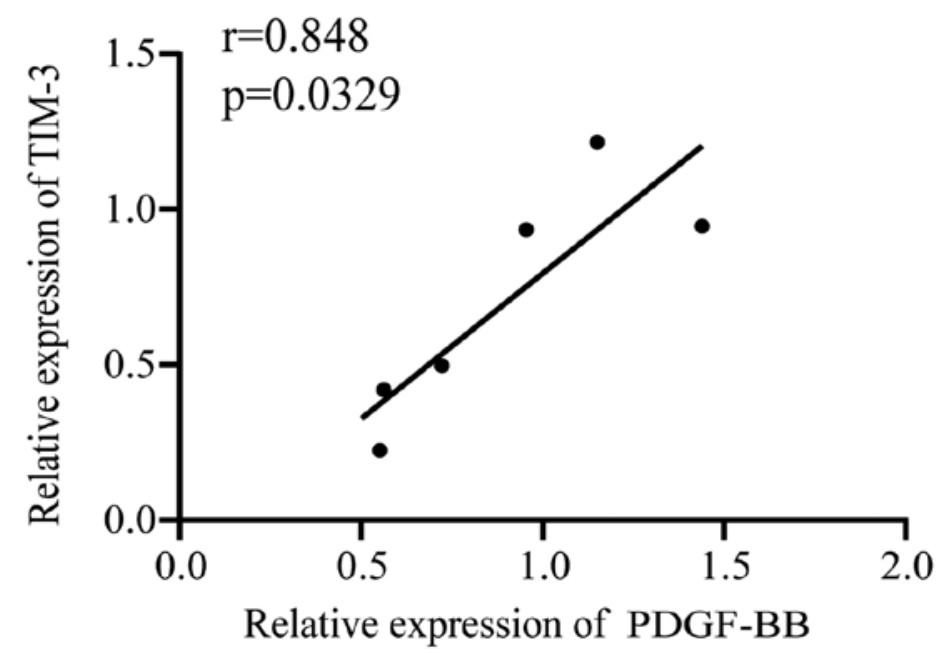

Figure 1. Differences in TIM-3 expression between LEAOD and normal arterial tissues. (A) Immunohistochemical analysis of TIM-3 expression in LEAOD and normal arterial tissues (magnification, $\mathrm{x} 400$ ). (B) TIM-2 and PDGF-BB protein expression levels in LEAOD and normal arterial tissues were determined by western blotting and quantification. (C) Correlation between the relative expression of PDGF-BB and TIM-3. ${ }^{*} \mathrm{P}<0.05,{ }^{* *} \mathrm{P}<0.01,{ }^{* * *} \mathrm{P}<0.001$. TIM-3, $\mathrm{T}-\mathrm{ce} 11$ immunoglobulin and mucin domain 3; LEAOD, lower extremity arteriosclerosis obliterans disease; PDGF-BB, platelet-derived growth factor-BB.

TIM-3 overexpression inhibits proliferation and migration in PDGF-BB-stimulated HASMCs. To further investigate the functional role of TIM-3, a lentiviral vector (LV-TIM-3) was used to overexpress TIM-3 in HASMCs (Fig. S1). To determine the effect of TIM-3 on the proliferation and migration of HASMCs, EdU (Fig. 3A and D), Transwell (Fig. 3B and E) and wound healing (Fig. 3C and F) assays were performed. The proportion of EdU-positive cells was significantly lower 
A

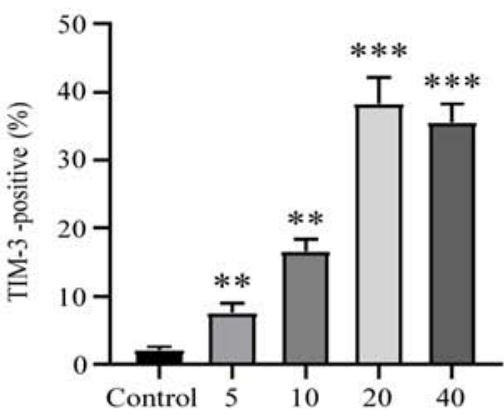

PDGF-BB (ng/ml)

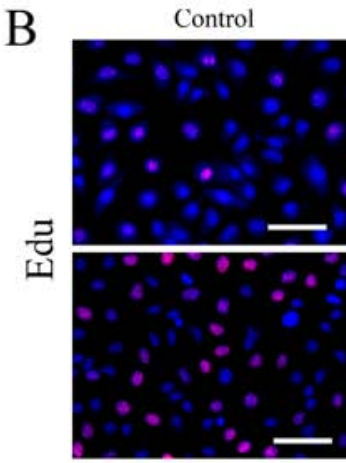

PDGF-BB+anti-TIM-3

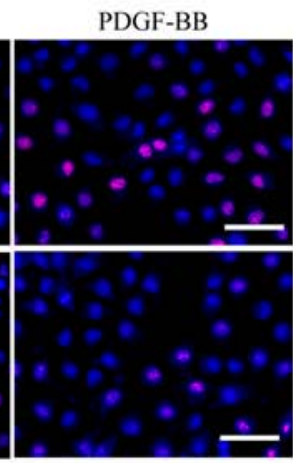

PDGF-BB+TIM-3

C
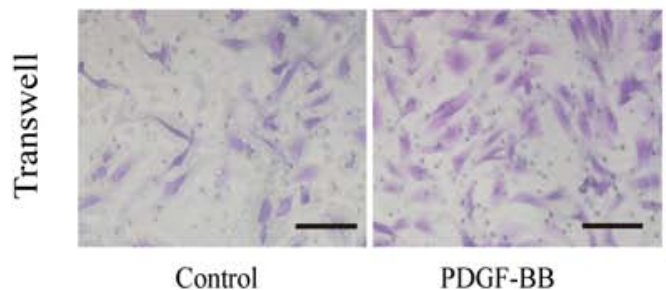

PDGF-BB

D

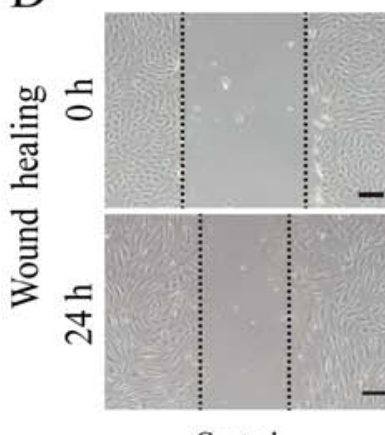

Control

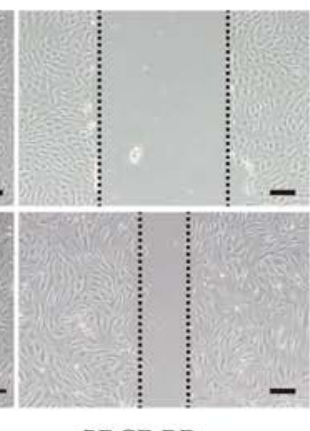

PDGF-BB

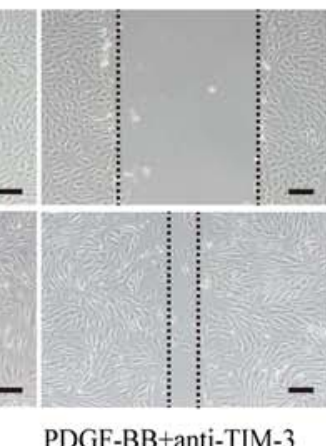

PDGF-BB+anti-TIM-3

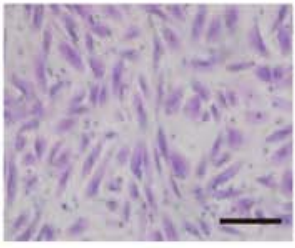

PDGF-BB+anti-TIM-3

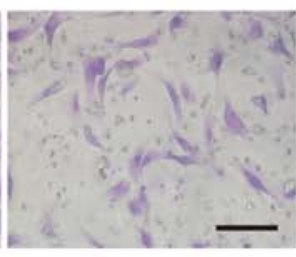

PDGF-BB+TIM-3
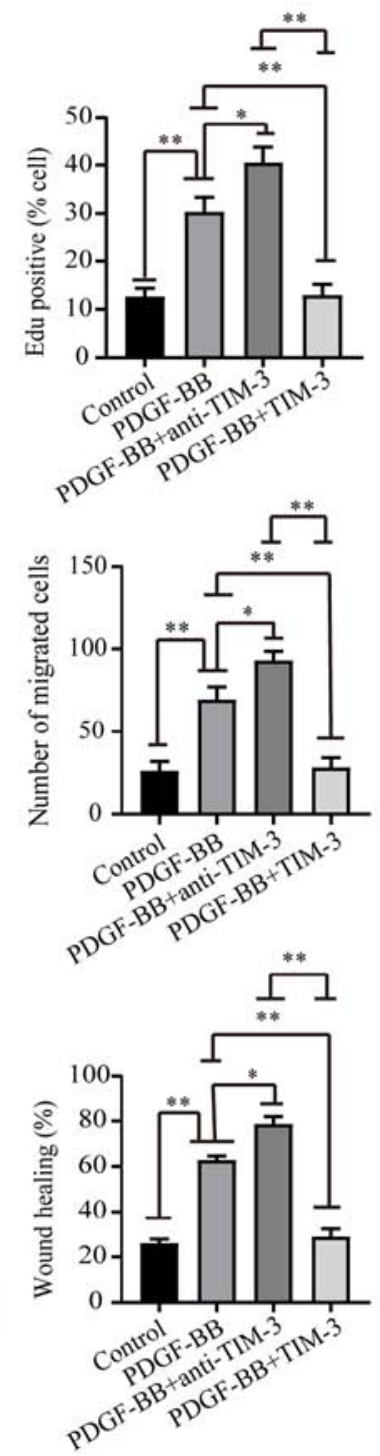

Figure 2. TIM-3 inhibits PDGF-BB-induced proliferation and migration in HASMCs. (A) Quantification of flow cytometric analysis of the proportion of TIM-3 ${ }^{+}$PDGF-BB-stimulated HASMCs. ${ }^{* *} \mathrm{P}<0.01$ and ${ }^{* * *} \mathrm{P}<0.001$ vs. control group (B) EdU, (C) Transwell and (D) wound healing assays were used to assess proliferation and migration in PDGF-BB-stimulated $(20 \mathrm{ng} / \mathrm{ml})$ HASMCs in the presence or absence of TIM-3 $(1,000 \mathrm{ng} / \mathrm{ml})$ and anti-TIM-3 $(10 \mu \mathrm{g} / \mathrm{ml} ; \mathrm{n}=5$; scale bar, $100 \mu \mathrm{m}) .{ }^{*} \mathrm{P}<0.05,{ }^{* *} \mathrm{P}<0.01$. TIM-3, T-cell immunoglobulin and mucin domain 3; PDGF-BB, platelet-derived growth factor-BB; HASMCs, human artery vascular smooth muscle cells.

in the PDGF-BB + LV-TIM-3 group (11.58 $\pm 3.36 \%)$ compared to the PDGF-BB $(29.42 \pm 3.72 \%)$ and the PDGF-BB + Vector $(31.18 \pm 7.78 \%)$ groups, but was not significantly different compared to the control group $(10.06 \pm 3.18 \%$; Fig. $3 \mathrm{~A})$. The mean number of migratory cells was significantly reduced in the PDGF-BB + LV-TIM-3 group (22.0 26.2$)$ compared to the PDGF-BB (49.0 \pm 9.8$)$ and PDGF-BB + Vector $(51.8 \pm 6.1)$ groups, but was also not significantly different compared to the control group (22.4 \pm 4.9 ; Fig. 3B). The wound healing assay results were similar to the Transwell assay results (Fig. 3C). The results demonstrated that LV-TIM-3-mediated TIM-3 overexpression in HASMCs inhibited proliferation and migration in PDGF-BB-stimulated HASMCs.

TIM-3 decreases IL- 6 and TNF- $\alpha$ expression. TIM-3 is an important inflammatory response regulator (16). To further investigate the role of TIM-3 in atherosclerosis, the expression of proinflammatory cytokines in PDGF-BB-stimulated
HASMCs treated with or without LV-TIM-3 was investigated. Western blot analysis indicated that the PDGF-BB group exhibited higher expression levels of IL- 6 and TNF- $\alpha$ compared with the control group. TIM-3 overexpression in HASMCs resulted in a significant decrease in IL- 6 and TNF- $\alpha$ expression compared to the PDGF-BB group (Fig. 4A and B). The results indicated that TIM-3 suppressed PDGF-BB-induced inflammatory responses by decreasing the expression levels of proinflammatory factors.

TIM-3 protects HASMCs from the PDGF-BB-induced proinflammatory response by inhibiting $N F-\kappa B$ signaling. The $\mathrm{NF}-\kappa \mathrm{B}$ signaling pathway is important for PDGF-BB-induced VSMC inflammatory factor secretion, which promotes proliferation and migration in VSMCs (22). To further investigate whether the NF- $\kappa$ B signaling pathway was related to TIM-3, the protein expression of members of the signaling pathway were assessed by western blotting in the control, PDGF-BB, 
A

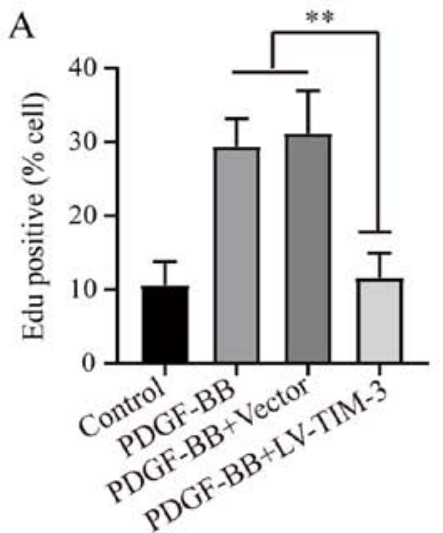

B

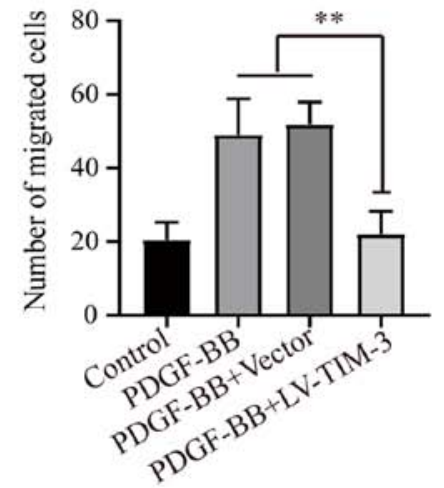

C

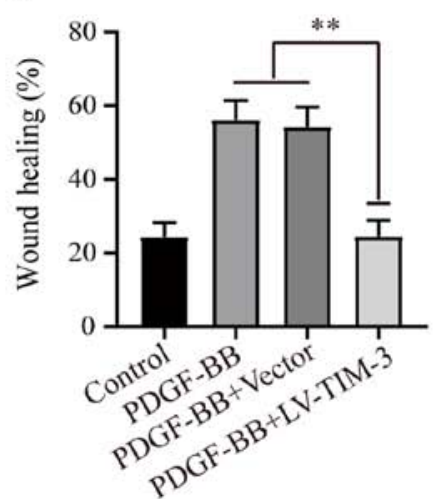

D
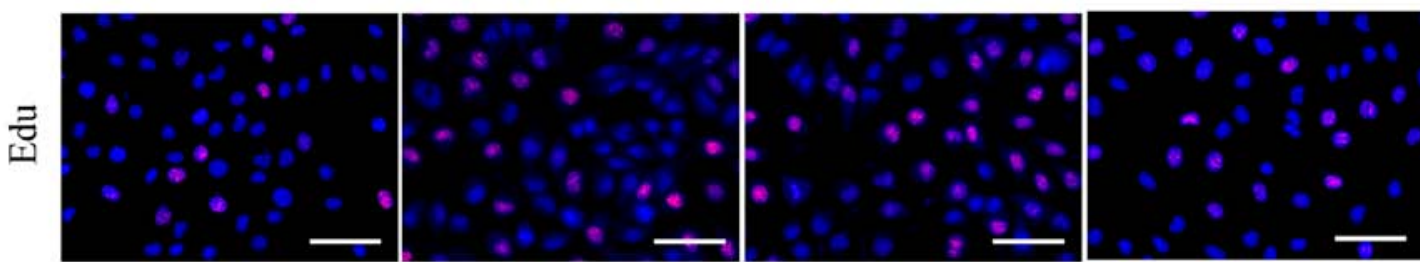

E

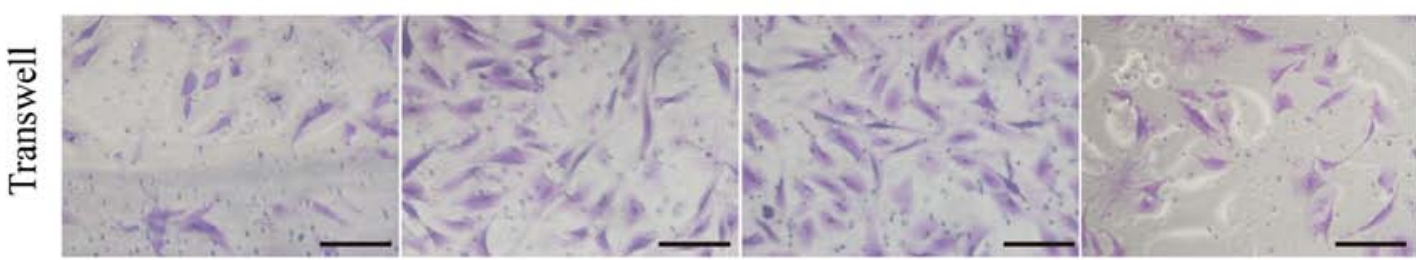

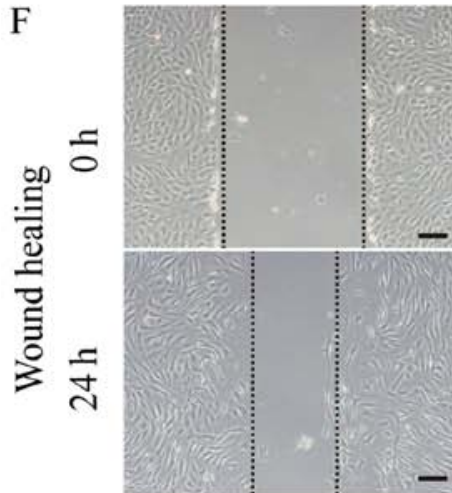

Control

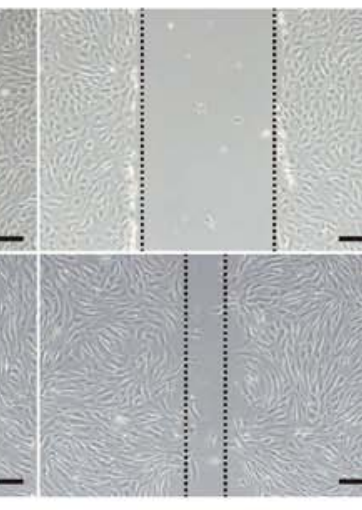

PDGF-BB

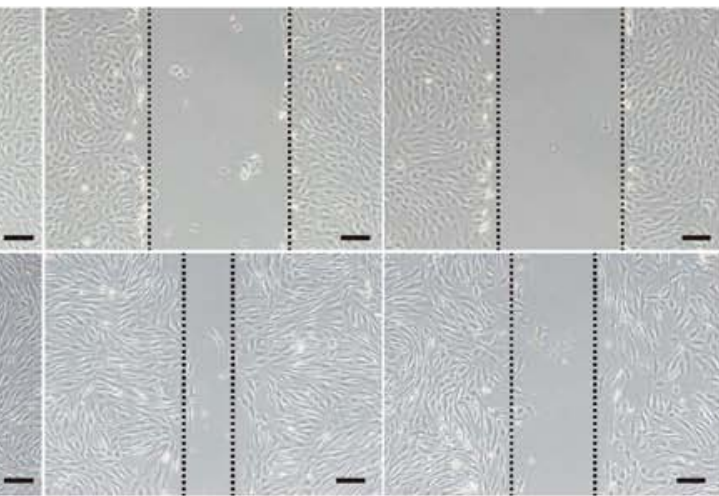

PDGF-BB+Vector PDGF-BB+LV-TIM-3

Figure 3. Effect of TIM-3 overexpression on proliferation and migration in PDGF-BB-stimulated HASMCs. (A) Proportion of EdU-positive cells. (B) Number of migratory cells. (C) Ratio of wound healing. (D) EdU, (E) Transwell and (F) wound healing assays were performed to assess proliferation and migration in PDGF-BB-stimulated (20 ng/ml) HASMCs in the presence or absence of LV-TIM-3 ( $\mathrm{n}=5$; scale bar, $100 \mu \mathrm{m}){ }^{* * *} \mathrm{P}<0.01$ as indicated. TIM-3, T-cell immunoglobulin and mucin domain 3; PDGF-BB, platelet-derived growth factor-BB; HASMCs, human artery vascular smooth muscle cells; LV, lentivirus.

PDGF-BB + Vector and PDGF-BB + LV-TIM-3 groups. The expression of TIM-3 and p-p65 NF- $\kappa \mathrm{B}$ was significantly increased in the PDGF-BB group compared to the control group, but the total p65 NF- $\kappa \mathrm{B}$ levels were not significantly altered (Fig. 4C). The results indicated that PDGF-BB treatment increased the expression of $\mathrm{p}-\mathrm{p} 65-\mathrm{NF}-\kappa \mathrm{B}$ but not total-NF- $\kappa \mathrm{B}$, and activated the $\mathrm{NF}-\kappa \mathrm{B}$ signaling pathway in HASMCs. Furthermore TIM-3 overexpression reversed the PDGF-BB-induced effects on p65 NF- $\mathrm{B}$.

\section{Discussion}

TIM-3 plays an important role in autoimmune and alloimmune diseases, immune tolerance and tumors $(23,24)$. Previous studies have reported that TIM-3 displays an antiatherosclerotic role during the generation and progression of atherosclerosis in traditional immune cells, including mononuclear macrophages, T cells and NK cells $(16,19)$; however, few studies have investigated the function of TIM-3 in non-classical immune cells. In the present study, TIM-3 expression was significantly increased in LEAOD arterial tissues compared to normal arterial tissues. The present study revealed that TIM-3 was expressed in HASMCs, which are non-classical immune cells, and was upregulated following PDGF-BB stimulation.

PDGF-BB initiates a number of biological processes by increasing the levels of various proinflammatory factors, including high motility group box 1 protein, IL-1, TNF- $\alpha$ and IL-6, and plays a key role in VSMC proliferation and migra- 
A

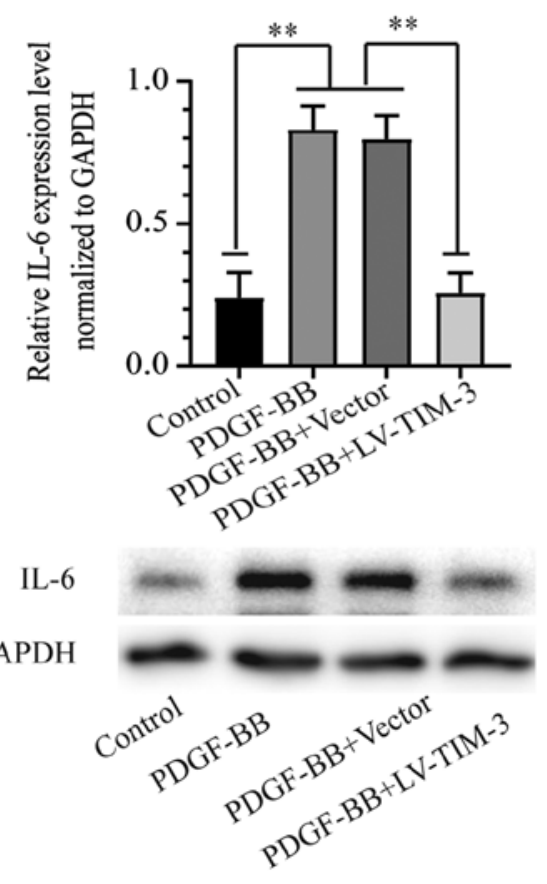

B

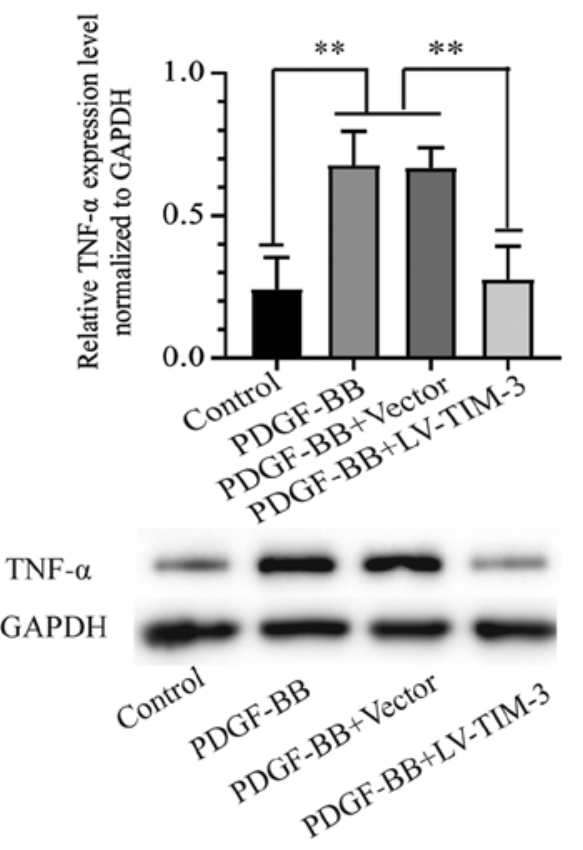

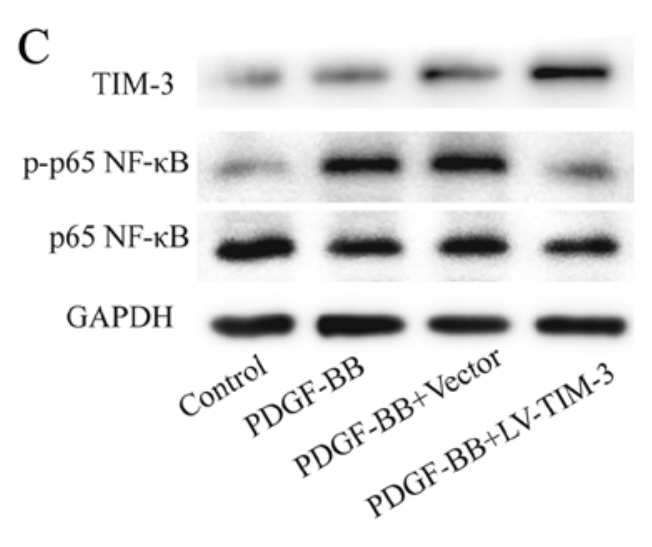
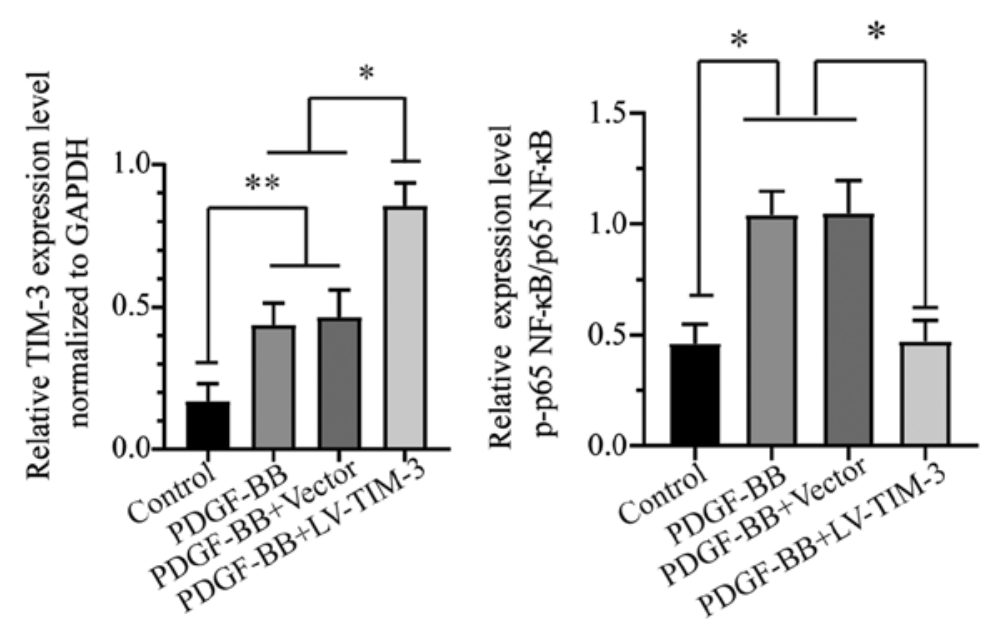

Figure 4.IL-6 and TNF- $\alpha$ expression in the four treatment groups and the effect of TIM- 3 overexpression on the NF- $\kappa$ B signaling pathway in PDGF-BB-stimulated

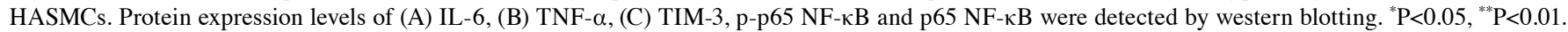
IL-6, interleukin-6; TNF- $\alpha$, tumor necrosis factor- $\alpha$; TIM-3, T-cell immunoglobulin and mucin domain 3; PDGF-BB, platelet-derived growth factor-BB; HASMCs, human artery vascular smooth muscle cells; p, phosphorylated; LV, lentivirus.

tion (25). Inhibiting VSMC proliferation and migration is an important therapeutic strategy for atherosclerosis (26-28). TIM-3 displays antiatherosclerotic effects during the development of atherosclerosis in traditional immune cells, but its effect on VSMC proliferation and migration has not been reported (16). In the present study, a positive correlation between the relative expression of PDGF-BB and TIM-3 in tissues was identified. The relationship may be due to the increased expression of PDGF-BB during atherogenesis, followed by the upregulation of inflammatory factors in VSMCs, such as IL-6 and TNF- $\alpha$. In the present study, VSMCs displayed an adaptive response against inflammation, and the expression of TIM-3, an immune tolerance factor, increased correspondingly.

In the present study, the expression of TIM-3 increased following PDGF-BB stimulation of HASMCs, and TIM-3 reduced $\mathrm{PDGF}-\mathrm{BB}$-induced migration and proliferation in HASMCs. Treatment of HASMCs with anti-TIM-3 reversed the effects of TIM-3. Therefore, it was hypothesized that TIM-3 displays increased adaptability to PDGF-BB, negatively regulates the PDGF-BB-induced inflammatory response, and inhibits migration and proliferation in HASMCs.

Subsequently, TIM-3 was overexpressed in HASMCs using LV-TIM-3 to investigate whether TIM-3 was a functional effector in HASMCs. TIM-3 overexpression inhibited proliferation and migration in PDGF-BB-stimulated HASMCs. Qiu et al (29) reported that TIM-3 inhibited ox-low density lipoprotein-induced atherogenic responses in HUVECs, which was consistent with the results of the present study, indicating that TIM-3 displays antiatherosclerotic effects.

The 'inflammation theory' and 'injury-response theory' have become mainstream theories for the pathogenesis of atherosclerosis $(30,31) . N F-\kappa \mathrm{B}$ is a transcription factor that is abundantly expressed in human atherosclerotic lesion VSMCs, macrophages and endothelial cells, and 
is associated with various signaling pathways involved in the inflammatory response, which induce atherosclerosis development $(32,33)$. The p50/p65 heterodimer is the most common $\mathrm{NF}-\kappa \mathrm{B} / \mathrm{Rel}$ proto-oncogene complex, which is present in the majority of cells in vivo. Increased p65 $\mathrm{NF}-\kappa \mathrm{B}$ phosphorylation often indicates activation of the $\mathrm{NF}-\kappa \mathrm{B}$ signaling pathway $(34,35)$. In the present study, the proinflammatory factors IL- 6 and TNF- $\alpha$ were expressed at high levels in PDGF-BB-stimulated HASMCs alongside increased levels of p $65 \mathrm{NF}-\kappa \mathrm{B}$ phosphorylation. The results indicated that $\mathrm{PDGF}-\mathrm{BB}$ stimulation activated the $\mathrm{NF}-\kappa \mathrm{B}$ signaling pathway and the expression of associated proinflammatory factors in VSMCs. In addition, based on the results of the present study, TIM-3 upregulation may serve as a self-regulatory mechanism of VSMCs against inflammation, but this induction may not be sufficient to counteract the proinflammatory effects of PDGF-BB. TIM-3 overexpression resulted in a significant decrease in the expression levels of proinflammatory factors and p65 $\mathrm{NF}-\kappa \mathrm{B}$ phosphorylation in HASMCs, which suggested that TIM-3 overexpression inhibited the activation of the NF- $\mathrm{B}$ signaling pathway and exerted an anti-inflammatory effect.

In previous studies, it has been reported that TIM-3 can inhibit the development of atherosclerosis $(16,17,29)$, which were similar to the results of the present study. Although the antiatherosclerotic effect of TIM-3 in non-classical immune cells was indicated in the present study, the underlying mechanisms were not identified. Therefore, further investigation is required to identify the mediators and factors underlying the results obtained in the present study, and to identify other signaling pathways that may mediate the effects of TIM-3 on HASMC proliferation and migration.

In the present study, protein arrays indicated that TIM-3 was upregulated in the serum of patients with LEAOD. Immunohistochemistry and western blotting of arterial tissue further revealed that TIM-3 expression was increased in LEAOD artery tissue compared with normal artery tissue. Furthermore, to the best of our knowledge, the present study revealed for the first time that TIM-3 inhibited proliferation and migration in PDGF-BB-induced HASMCs by inhibiting HASMC inflammatory responses.

In conclusion, TIM-3 decreased the proliferation and migration of PDGF-BB-induced HASMCs and downregulated the expression of proinflammatory factors by inhibiting the NF- $\kappa \mathrm{B}$ signaling pathway. The results suggested that TIM-3 may serve as a protective factor against inflammation and atherogenic responses in HASMCs. Furthermore, TIM-3 may serve as a potential target for the prevention and treatment of atherosclerosis.

\section{Acknowledgements}

The authors would like to thank Dr Lei Zhao and Dr Jin Cui (The First Affiliated Hospital of Sun Yat-sen University) for their editorial support.

\section{Funding}

The present study was supported by the National Natural Science Foundation of China (grant no. 81873813).

\section{Availability of data and materials}

The datasets used and during the present study are available from the corresponding author upon reasonable request.

\section{Authors' contributions}

CL conceived the study, performed the experiments and wrote the manuscript. ZCW, JCQ and BHJ drafted the work, and collected and analyzed the data. JBL and RZH contributed to the interpretation of the data. RML and WL designed and drafted the work. SMW and JSW conceived the experiments and revised the manuscript for important intellectual content. All authors read and approved the final manuscript.

\section{Ethics approval and consent to participate}

The present study was approved by the Ethics Committee of the First Affiliated Hospital of Sun Yat-sen University (approval no. 151507). Written informed consent was obtained from the participants or their relatives.

\section{Patient consent for publication}

Not applicable.

\section{Competing interests}

The authors declare that they have no competing interests.

\section{References}

1. Criqui MH, Vargas V, Denenberg JO, Ho E, Allison M, Langer RD, Gamst A, Bundens WP and Fronek A: Ethnicity and peripheral arterial disease: The San Diego Population Study. Circulation 112: 2703-2707, 2005.

2. Fowkes FG, Rudan D, Rudan I, Aboyans V, Denenberg JO, McDermott MM, Norman PE, Sampson UK, Williams LJ, Mensah GA and Criqui MH: Comparison of global estimates of prevalence and risk factors for peripheral artery disease in 2000 and 2010: A systematic review and analysis. Lancet 382: 1329-1340, 2013.

3. Crotty S: T Follicular Helper cell biology: A decade of discovery and diseases. Immunity 50: 1132-1148, 2019.

4. Yu E, Hsu HY, Huang CY and Hwang LC: Inflammatory biomarkers and risk of atherosclerotic cardiovascular disease. Open Med (Wars) 13: 208-213, 2018.

5. Kim J, Zhang L, Peppel K, Wu JH, Zidar DA, Brian L, DeWire SM, Exum ST, Lefkowitz RJ and Freedman NJ: Beta-arrestins regulate atherosclerosis and neointimal hyperplasia by controlling smooth muscle cell proliferation and migration. Circ Res 103: 70-79, 2008.

6. Lee $\mathbf{J}$ and Kang H: Hypoxia Promotes vascular smooth muscle cell proliferation through microRNA-mediated suppression of Cyclin-dependent kinase inhibitors. Cells 8: pii: E802, 2019.

7. Heldin CH and Westermark B: Mechanism of action and in vivo role of platelet-derived growth factor. Physiol Rev 79: 1283-1316, 1999.

8. Liu K, Liu C and Zhang Z: IncRNA GAS5 acts as a ceRNA for miR-21 in suppressing PDGF-bb-induced proliferation and migration in vascular smooth muscle cells. J Cell Biochem 120: 15233-15240, 2019.

9. $\mathrm{Hu} \mathrm{W}$ and Huang Y: Targeting the platelet-derived growth factor signalling in cardiovascular disease. Clin Exp Pharmacol Physiol 42: 1221-1224, 2015.

10. Zofková I: Osteoporosis and aterosclerosis-is there any pathogenetic association? Cas Lek Cesk 146: 246-250, 2007 (In Czech).

11. Hartman J and Frishman WH: Inflammation and atherosclerosis: A review of the role of interleukin- 6 in the development of atherosclerosis and the potential for targeted drug therapy. Cardiol Rev 22: 147-151, 2014. 
12. Song C, Wang Y, Cui L, Yan F and Shen S: Triptolide attenuates lipopolysaccharide-induced inflammatory responses in human endothelial cells: Involvement of $\mathrm{NF}-\kappa \mathrm{B}$ pathway. BMC Complement Altern Med 19: 198, 2019.

13. Ponte E and Ursu HI: Overt and subclinical hypothyroidism and atherosclerotic arteriopathy of the lower limbs (clinical and subclinical). Rom J Endocrinol 31: 71-79, 1993.

14. Hou N, Zhao D, Liu Y, Gao L, Liang X, Liu X, Gai X, Zhang X Zhu F, Ni M, et al: Increased expression of $\mathrm{T}$ cell immunoglobulin- and mucin domain-containing molecule-3 on natural killer cells in atherogenesis. Atherosclerosis 222: 67-73, 2012.

15. Liang X, Xu Z, Yuan M, Zhang Y, Zhao B, Wang J, Zhang A and Li G: MicroRNA-16 suppresses the activation of inflammatory macrophages in atherosclerosis by targeting PDCD4. Int J Mol Med 37: 967-975, 2016.

16. Foks AC, Ran IA, Wasserman L, Frodermann V, Ter Borg MN, de Jager SC, van Santbrink PJ, Yagita H, Akiba H, Bot I, et al T-cell immunoglobulin and mucin domain 3 acts as a negative regulator of atherosclerosis. Arterioscler Thromb Vasc Biol 33: 2558-2565, 2013

17. Qiu MK, Wang SC, Dai YX, Wang SQ, Ou JM and Quan ZW: PD-1 and Tim-3 pathways regulate CD8+ T cells function in atherosclerosis. PLoS One 10: e0128523, 2015.

18. Hu W, Wang M, Yin H, Yao C, He Q, Yin L, Zhang C, Li W, Chang $\mathrm{G}$ and Wang S: MicroRNA-1298 is regulated by DNA methylation and affects vascular smooth muscle cell function by targeting connexin 43. Cardiovasc Res 107: 534-545, 2015.

19. Zhang N, Zhang M, Liu RT, Zhang P, Yang CL, Yue LT, Li H, Li YK and Duan RS: Statins reduce the expressions of Tim-3 on NK cells and NKT cells in atherosclerosis. Eur J Pharmacol 821: 49-56, 2018

20. Zhang F, Zhao J, Sun D and Wei N: miR-155 inhibits transformation of macrophages into foam cells via regulating $\mathrm{CEH}$ expression. Biomed Pharmacother 104: 645-651, 2018.

21. Bennett MR, Sinha S and Owens GK: Vascular smooth muscle cells in atherosclerosis. Circ Res 118: 692-702, 2016.

22. Maracle CX, Agca R, Helder B, Meeuwsen JAL, Niessen HWM, Biessen EAL, de Winther MPJ, de Jager SCA, Nurmohamed MT and Tas SW: Noncanonical NF- $\mathrm{KB}$ signaling in microvessels of atherosclerotic lesions is associated with inflammation, atheromatous plaque morphology and myocardial infarction. Atherosclerosis 270: 33-41, 2018.

23. Das M, Zhu C and Kuchroo VK: Tim-3 and its role in regulating anti-tumor immunity. Immunol Rev 276: 97-111, 2017.

24. Sánchez-Fueyo A, Tian J, Picarella D, Domenig C, Zheng XX, Sabatos CA, Manlongat N, Bender O, Kamradt T, Kuchroo VK, et al: Tim-3 inhibits T helper type 1-mediated auto- and alloimmune responses and promotes immunological tolerance. Nat Immunol 4: 1093-1101, 2003.
25. Huang SC, Wang M, Wu WB, Wang R, Cui J, Li W, Li ZL, Li W and Wang SM: miR-22-3p inhibits arterial smooth muscle cell proliferation and migration and neointimal hyperplasia by targeting HMGB1 in arteriosclerosis obliterans. Cell Physio Biochem 42: 2492-2506, 2017.

26. Andrés V: Control of vascular cell proliferation and migration by cyclin-dependent kinase signalling: New perspectives and therapeutic potential. Cardiovasc Res 63: 11-21, 2004

27. Zhang Y, Qian X, Sun X, Lin C, Jing Y, Yao Y, Ma Z, Kuai M, Lu Y, Kong X, et al: Liuwei Dihuang, a traditional Chinese medicinal formula, inhibits proliferation and migration of vascular smooth muscle cells via modulation of estrogen receptors. Int J Mol Med 42: 31-40, 2018

28. Hao B, Xiao Y, Song F, Long X, Huang J, Tian M, Deng S and Wu Q: Metformin-induced activation of AMPK inhibits the proliferation and migration of human aortic smooth muscle cells through upregulation of p53 and IFI16. Int J Mol Med 41: 1365-1376, 2018

29. Qiu MK, Wang SC, Tang Y, Pan C, Wang Y, Wang SQ, Quan ZW and Ou JM: Tim-3 inhibits low-density lipoprotein-induced atherogenic responses in human umbilical vein endothelial cells. Oncotarget 8: 61001-61010, 2017.

30. Ohira H, Tsutsui W and Fujioka Y: Are Short Chain Fatty Acids in Gut Microbiota defensive players for inflammation and atherosclerosis. J Atheroscler Thromb 24: 660-672, 2017.

31. Pant S, Deshmukh A, Gurumurthy GS, Pothineni NV, Watts TE, Romeo F and Mehta JL: Inflammation and atherosclerosis-revisited. J Cardiovasc Pharmacol Ther 19: 170-178, 2014.

32. Gliozzi M, Scicchitano M, Bosco F, Musolino V, Carresi C, Scarano F, Maiuolo J, Nucera S, Maretta A, Paone S, et al: Modulation of nitric oxide synthases by oxidized LDLs: Role in vascular inflammation and atherosclerosis development. Int J Mol Sci 20: pii: E3294, 2019

33. Pan JX: LncRNA H19 promotes atherosclerosis by regulating MAPK and NF- $\kappa$ B signaling pathway. Eur Rev Med Pharmacol Sci 21: 322-328, 2017.

34. Ge H, Tang H, Liang Y, Wu J, Yang Q, Zeng L and Ma Z: Rhein attenuates inflammation through inhibition of NF- $\kappa B$ and NALP3 inflammasome in vivo and in vitro. Drug Des Devel Ther 11: 1663-1671, 2017.

35. Lai JL, Liu YH, Liu C, Qi MP, Liu RN, Zhu XF, Zhou QG, Chen YY, Guo AZ and Hu CM: Indirubin inhibits LPS-induced inflammation via TLR4 abrogation mediated by the NF- $\kappa B$ and MAPK signaling pathways. Inflammation 40: 1-12, 2017.

This work is licensed under a Creative Commons Attribution-NonCommercial-NoDerivatives 4.0 International (CC BY-NC-ND 4.0) License. 